\title{
Subdiagnóstico de leptospirosis en el Valle del Cauca: ¿La punta del iceberg?
}

\section{Under-diagnosis of leptospirosis in the Cauca Valley: The tip of the iceberg?}

\author{
Andrés Augusto González ${ }^{1}$, Sebastián Baena ${ }^{1}$, Durney Palomino², Beatriz E. Ferro ${ }^{1}$, \\ Robinson Pacheco ${ }^{1}$ \\ ${ }^{1}$ Universidad Icesi, Facultad de Ciencias de la Salud, Cali, Colombia \\ ${ }^{2}$ Laboratorio Departamental de Salud Pública del Valle del Cauca, Cali, Colombia
}

\begin{abstract}
Resumen
La leptospirosis es la zoonosis bacteriana más frecuente. En Colombia la prevalencia alcanza hasta $35 \%$ (1); el Valle del Cauca reporta alrededor del $23 \%$ de los casos. Las pruebas de laboratorio no son ampliamente disponibles, lo que lleva al subdiagnóstico y subregistro. El objetivo fue describir las características clínico-demográficas y el cumplimiento del proceso de notificación de los casos, remitidos al laboratorio departamental de salud pública del Valle del Cauca entre 2017 y 2018.

Se trata de un estudio observacional, descriptivo y retrospectivo. Se incluyen los casos con sospecha clínica y/o paraclínica que fueron notificados al Sistema Nacional de Vigilancia en Salud Pública (Sivigila) y que estuvieran incluidos en la base de datos del Laboratorio Departamental del Valle del Cauca entre 2017 y 2018.

Se evaluaron 256 registros, de los cuales $41,4 \%$ tuvieron prueba de ELISA positiva. El análisis final se hizo con 105 casos. La mediana de edad fue de 33 años (RIC: 19 - 53), 67,62\% de sexo masculino. La mayoría se reportaron en Cali $(34,29 \%)$. La mediana de días de síntomas fue de 6 (RIC: $4-8)$, con la fiebre como el más frecuente $(92,38 \%$ ). Solo en $51,43 \%$ hubo cumplimiento de la prueba de tamizaje en la institución prestadora de salud, sin embargo, 38,89\% tenían resultado negativo para leptospirosis a pesar del resultado positivo de la prueba ELISA en el Laboratorio Departamental.

Los resultados de esta investigación coinciden con estudios previos. Continúa siendo una entidad frecuente en el departamento con dificultad en el diagnóstico y notificación. Es necesario fortalecer la capacidad diagnóstica y el cumplimiento de la notificación.
\end{abstract}

Palabras clave: Leptospirosis; Leptospira; Colombia; zoonosis; Vigilancia en salud pública. 


\begin{abstract}
Leptospirosis is the most common bacterial zoonosis. In Colombia the prevalence reaches up to $35 \%$ (1); Valle del Cauca reports about $23 \%$ of the cases. Laboratory tests are not widely available, leading to underdiagnosis and underreporting. The objective was to describe the clinicaldemographic characteristics and compliance with the notification process of the cases, sent to the departmental public health laboratory of Valle del Cauca between 2017 and 2018.

It is an observational, descriptive and retrospective study. Cases with clinical and/or paraclinical suspicion that were notified to the National Public Health Surveillance System (Sivigila) and that were included in the database of the Valle del Cauca Departmental Laboratory between 2017 and 2018 are included.

256 records were evaluated, of which $41.4 \%$ had a positive ELISA test. The final analysis was done on 105 cases. The median age was 33 years (IQR: $19-53), 67.62 \%$ male. Most were reported in Cali (34.29\%). The average time length of symptoms was 6 days (IQR: 4-8). Fever was the most frequent $(92.38 \%)$. Only $51.43 \%$ had compliance with the screening test in the health provider institution, however, $38.89 \%$ had a negative result for leptospirosis despite the positive result of the ELISA test in the Departmental Laboratory.

The results of this research coincide with previous studies. The difficulty in diagnosis and notification in the department continues to be a frequent phenomenon. It is necessary to strengthen the capacity for diagnosis, compliance and notification.
\end{abstract}

Keywords: Leptospirosis; Leptospira; Colombia; zoonosis; Public health surveillance.

\section{Introducción}

La leptospirosis es la zoonosis bacteriana más frecuentemente reportada en humanos; aunque tiene distribución mundial, la mayoría de los reportes suceden en países tropicales (2). El agente etiológico pertenece al género Leptospira, cuyos serovares patógenos se agrupan dentro del complejo interrogans (3)(4).

Aunque se han reportado diversos factores de riesgos demográficos, ambientales y sociales para la infección, la principal exposición es ocupacional, asociada a labores agrícolas y pecuarias en las cuales hay exposición a desechos o derivados de animales reservorios de la espi- roqueta. Otros factores que potencian el riesgo de infección son el género masculino, el analfabetismo y determinantes relacionados con la extrema pobreza, entre los que se reportan necesidades básicas insatisfechas, cercanía de la vivienda a aguas residuales, ausencia de acueducto y alcantarillado, pisos de tierra y exposición a roedores (5)(6). Por otro lado, se sabe que la incidencia de la enfermedad aumenta en los periodos más lluviosos y en eventos catastróficos como inundaciones, cuando la exposición a aguas contaminadas es más frecuente. En países desarrollados, la exposición a aguas contaminadas es de tipo recreativa (7). 
Las manifestaciones clínicas de la leptospirosis son amplias e inespecíficas. La enfermedad puede cursar en su estado más benigno como un cuadro de fiebre de origen desconocido y autolimitado, el cual no se reporta al sistema de vigilancia epidemiológica; o puede cursar como una enfermedad severa y hasta causar la muerte (8). La ausencia de signos y síntomas patognomónicos requiere que los casos sean confirmados a través de pruebas de laboratorio. En las áreas rurales de países en desarrollo, como Colombia, donde la infección es más frecuente, la disponibilidad de pruebas diagnósticas de alto rendimiento es limitada, por ello, los casos no se configuran o se reportan a los sistemas de vigilancia como casos sospechosos; esto explica el subdiagnóstico y subregistro en la notificación de la enfermedad. La disponibilidad de herramientas diagnosticas se agrupa en centros especializados o laboratorios de salud pública, donde se tienen técnicas de procesamiento de ELISA indirecta, donde se utilizan tiras de pozos reactivas, recubiertas con el antígeno (fase solida). Los anticuerpos específicos presentes en la muestra del paciente se ligan a estos antígenos de la fase sólida. Este test es cualitativo para la detección de anticuerpos humanos de género específico contra las Leptospira en suero y su utilidad está dada en los pacientes con fase aguda de la enfermedad, también se utilizan pruebas moleculares; sin embargo, la prueba o estándar de oro es el MAT (MAT - Microscopic agglutination test), que evalúa la reacción de anticuerpos plasmáticos contra antígenos del microorganismo, para esto se utilizan muestras de suero pareadas (tomada la primera al inicio de síntomas y la segunda de 10 a 15 días después de la toma de la primera muestra) se usa para la confirmación de la vigilan- cia en Salud Pública por parte del Instituto Nacional de Salud (9)(8).

Anualmente se reportan alrededor de 1,03 millones de casos en todo el mundo, con una incidencia que varía entre 0,1 y 975 casos por cada 100000 habitantes, mientras que la letalidad se calcula en $6,85 \%$ (10). La enfermedad representa una carga global de 2,9 millones de DALYs (Disability Adjusted Life Years) por año (11). En Colombia la prevalencia de la enfermedad ha sido tan alta como del $35 \%$ (1); el Departamento del Valle del Cauca reporta alrededor del $23 \%$ de los casos del país y se le considera como un territorio de alta prevalencia (12)(1). Algunos estudios realizados en la región se han enfocado en Cali y han reportado como principales factores de riesgo el contacto con roedores y otros mamíferos, además del contacto con aguas contaminadas (13), pero no se ha revisado recientemente el panorama del departamento y el proceso de notificación desde las unidades primarias generadora de datos hasta el nivel central (14)(15). El objetivo de esta investigación fue describir las características clínico-demográficas y el cumplimiento del proceso de notificación de los casos con sospecha de leptopirosis, remitidos al laboratorio departamental de salud pública del Valle del Cauca entre 2017 y 2018.

\section{Materiales y Métodos}

\subsection{Diseño del estudio}

Se realizó un estudio observacional descriptivo de serie de casos, con recolección retrospectiva de la información. 
González et al

\subsection{Población de referencia}

Se definieron como criterios de inclusión a todos los registros de pacientes atendidos en centros de salud del Valle del Cauca con sospecha clínica y/o paraclínica con prueba serológica rápida positiva, quienes fueron notificados y reportados al Sistema Nacional de Vigilancia en Salud Pública (Sivigila) a través de la ficha de notificación \# 455 y que estuvieran incluidos en la base de datos del Laboratorio Departamental del Valle del Cauca entre 2017 y 2018.

\subsection{Manejo de datos y análisis es- tadístico}

Toda la información clínica y demográfica se recolectó usando como documentos fuente a las fichas de notificación epidemiológica y de las historias clínicas que reposaban en el Laboratorio departamental de Salud Pública del Valle del Cauca en hojas de cálculo de Microsoft Office Excelßy analizada usando el paquete estadístico Stata 12 TM (Stata Corp, College Station, TX, USA). Las características demográficas y clínicas se resumieron a través de estadística descriptiva. El contraste de la normalidad de las variables numéricas se realizó a través de la prueba Shapiro Wilk, todas las variables se presentaron a través de la mediana y los rangos intercuartílicos. Las variables categóricas se presentaron como porcentajes. Para describir el cumplimiento del proceso de notificación al Sivigila, se calcularon los porcentajes en cada fase.

\subsection{Consideraciones éticas}

Este estudio fue avalado por el Comité de Ética de la Universidad Icesi de Cali, como una investigación sin riesgo de acuerdo a la Resolución 8430, según el acta Ndeg. 202 de 2018.

\section{Resultados}

Entre el primero de enero de 2017 y el 31 de diciembre de 2018, se evaluaron 256 registros de casos sospechosos de leptospirosis ocurridos en el Valle del Cauca los cuales fueron reportados al Sivigila. De esos registros, 106 (41,4\%) tuvieron prueba de ELISA positiva, realizada en el laboratorio departamental. En la base de datos no se obtuvo información de 1 paciente, por lo que el análisis final se realizó con 105 registros (Figura 1).

La mediana de edad de los pacientes fue de 33 años (RIC: 19 - 53). De estos pacientes, $71(67,62 \%)$ son de sexo masculino. El mayor número de casos se reportó en la ciudad de Cali con 36 registros $(34,29 \%)$, seguido por Tuluá con $26(24,76 \%)$ y Buga con 24 $(22,86 \%)$ (Tabla 1). La ocupación más reportada fue oficios varios, correspondiente a 38 casos $(36,19 \%)$, seguido de trabajo en el sector agropecuario con 33 casos $(31,43 \%)$. Se reportaron 73 pacientes $(70,19 \%)$ residentes en zona urbana. Del total de casos, $83(79,81 \%)$ refirió haber tenido algún contacto con animales, mientras que $46(43,81 \%)$ precisaron contacto con roedores. La disponibilidad de acueducto en la residencia se reportó en 85 casos $(81,73)$ (Tabla 1).

En cuanto a las características clínicas, la mediana de días de síntomas hasta el momento de la atención fue de 6 (RIC: 4 -8). El síntoma 


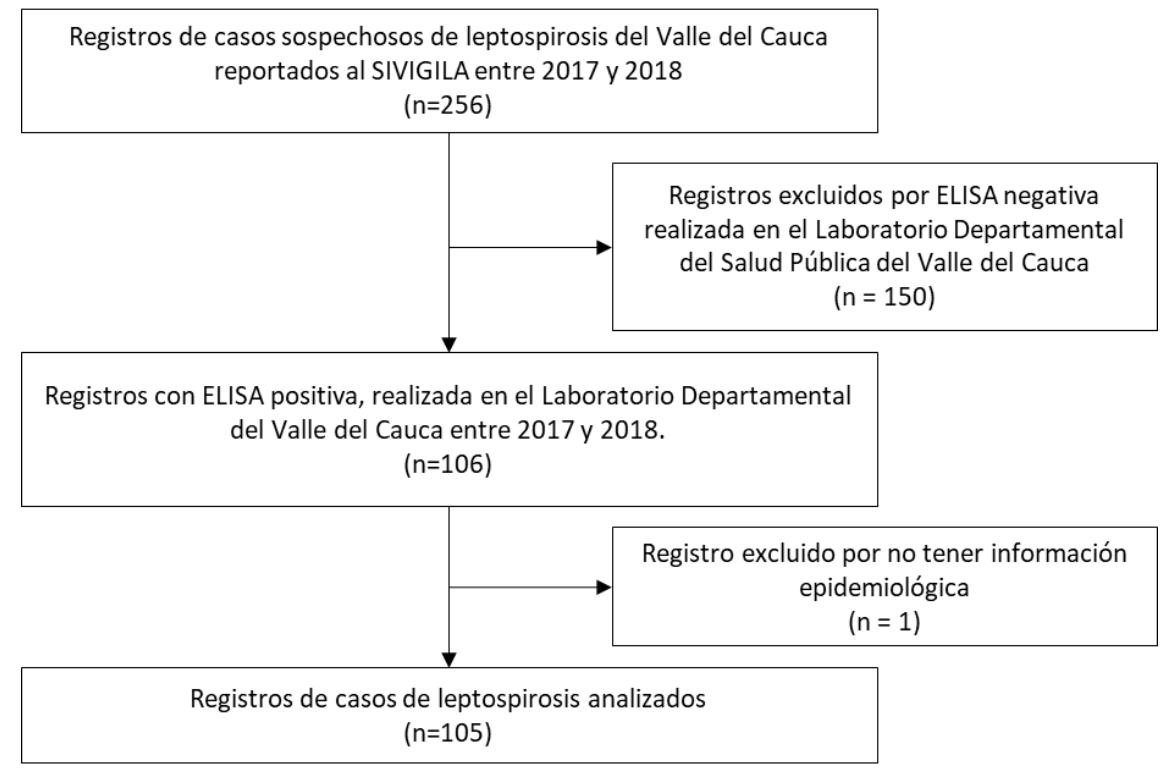

Figura 1: Diagrama de selección de la población de estudio.

más prevalente reportado fue la fiebre en 97 casos $(92,38 \%)$. Por otro lado, las mialgias se reportaron en 77 casos $(73,33 \%)$, seguido de cefalea en 61 casos $(58,10 \%)$, ictericia en 33 casos $(31,43 \%)$ y hepatomegalia en 13 casos $(12,38 \%)$ (Tabla 2).

De los 105 casos analizados, solo en 54 $(51,43 \%)$ se evidenció el cumplimiento de la realización de la prueba de tamiz para la detección de anticuerpos en la institución prestadora de servicios de salud. Todos los casos notificados contaron al menos con una muestra remitida al laboratorio Departamental de Salud Pública. De los 54 casos a los que se les documento la prueba tamiz, 21 (38,89\%) tenían resultado negativo para leptospirosis; sin embargo, la prueba de ELISA resultó positiva. El $100 \%$ de las muestras con prueba de ELISA positiva fue remitido al INS para realizar la prueba de MAT.
Respecto al comportamiento temporal de los casos reportados, durante el 2017 se presentó un pico en el mes de marzo con 12 casos. Durante el 2018, el mes con mayor reporte fue mayo con 14 casos, seguido de marzo con 11 casos (Figura 2).

\section{Discusión}

Este estudio describe las características clínico-demográficas y el cumplimiento del proceso de notificación de los casos con sospecha de leptopirosis remitidos al laboratorio departamental de salud pública del Valle del Cauca, entre 2017 y 2018.

La frecuencia de prueba de ELISA positiva para Leptospira, por parte del laboratorio Departamental, del $41,4 \%$ es cercana al $40 \%$ de confirmación de casos sospechosos por el laboratorio (12). Otros estudios realizados en 


\begin{tabular}{|c|c|c|c|}
\hline Característica & Descripción & $\mathrm{n}$ & Medida de Resumen \\
\hline Edad & años & 105 & *33 $(19-53)$ \\
\hline \multirow[t]{2}{*}{ Sexo } & Masculino & 71 & $67,62 \%$ \\
\hline & Femenino & 34 & $32,38 \%$ \\
\hline \multirow[t]{7}{*}{ Procedencia } & Cali & 36 & $34,29 \%$ \\
\hline & Tuluá & 26 & $24,76 \%$ \\
\hline & Buga & 24 & $22,86 \%$ \\
\hline & Palmira & 7 & $6,67 \%$ \\
\hline & Roldanillo & 6 & $5,71 \%$ \\
\hline & Alcalá & 4 & $3,81 \%$ \\
\hline & Otro & 2 & $1,90 \%$ \\
\hline \multirow[t]{5}{*}{ Ocupación } & Agropecuario & 33 & $31,43 \%$ \\
\hline & Estudiante & 20 & $19,05 \%$ \\
\hline & Hogar & 11 & $10,48 \%$ \\
\hline & Profesional & 3 & $2,86 \%$ \\
\hline & Oficios varios & 38 & $36,19 \%$ \\
\hline \multirow[t]{2}{*}{ Ocupación de riesgo } & NO & 80 & $76,19 \%$ \\
\hline & SI & 25 & $23,81 \%$ \\
\hline \multirow[t]{2}{*}{ Características de la zona } & Urbano & 73 & $70,19 \%$ \\
\hline & Rural & 32 & $29,81 \%$ \\
\hline \multirow[t]{2}{*}{ Contacto con animales* } & NO & 21 & $20,19 \%$ \\
\hline & SI & 83 & $79,81 \%$ \\
\hline \multirow[t]{2}{*}{ Contacto con ratas } & NO & 59 & $56,19 \%$ \\
\hline & SI & 46 & $43,81 \%$ \\
\hline \multirow[t]{2}{*}{ Acueducto } & $\mathrm{SI}$ & 85 & $81,73 \%$ \\
\hline & NO & 20 & $18,27 \%$ \\
\hline
\end{tabular}

Tabla 1: Caracterìsticas demográficas de pacientes con diagnóstico de leptospirosis reportados en el Valle del Cauca, 2017-2018.

Colombia han informado porcentajes de confirmación entre $12,2 \%$ (15)(13) y 31,3\% (1). Esta variabilidad en la confirmación de la infección de los casos sospechosos puede ser explicada por el tipo de prueba diagnóstica utilizada. En aquellos estudios con menor porcen- taje de confirmación se usó el MAT, mientras que en el presente estudio se utilizó prueba serológica, con menor rendimiento respecto al Gold standard.

Con relación a las características sociodemográficas, los hallazgos de este estudio están 


\begin{tabular}{llll}
\hline Característica & Descripción & N & Resumen \\
\hline Días de síntomas & Días & 105 & $* 6(4-8)$ \\
Fiebre & NO & 8 & $7,62 \%$ \\
Cefalea & SI & 97 & $92,38 \%$ \\
& NO & 44 & $41,90 \%$ \\
Mialgias & SI & 61 & $58,10 \%$ \\
& NO & 28 & $26,67 \%$ \\
Ictericia & SI & 77 & $73,33 \%$ \\
\hline \multirow{2}{*}{ Hepatomegalia } & NO & 72 & $68,57 \%$ \\
& SI & 33 & $31,43 \%$ \\
\hline
\end{tabular}

Tabla 2: Características clínicas de pacientes con diagnóstico de leptospirosis reportados en el Valle del Cauca, 2017-2018.

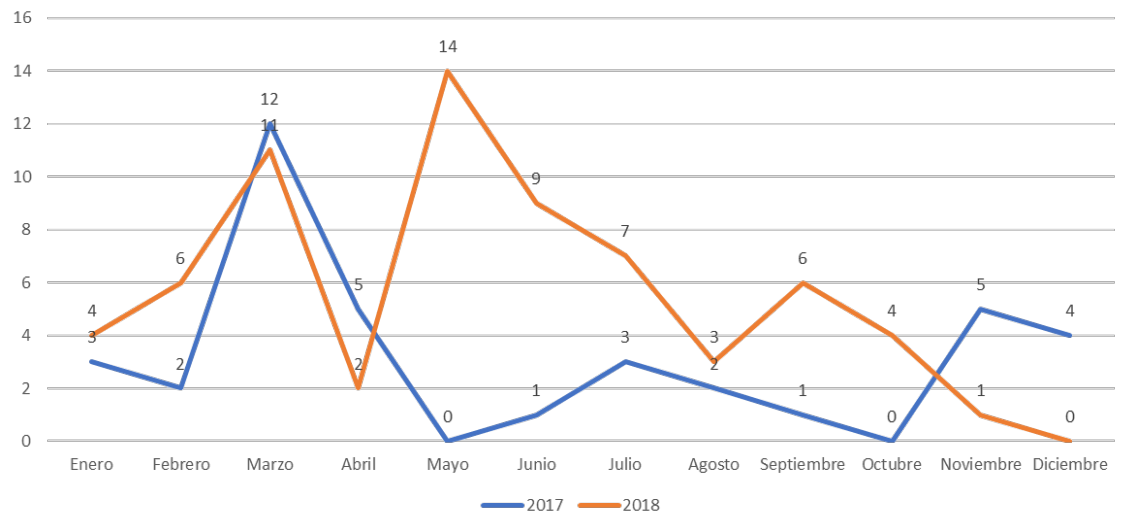

Figura 2: Comportamiento termporal de los casos de leptospirosis en el Valle del Cauca, 2017-2018.

alineados con lo reportado en otras investigaciones en el territorio nacional, en donde se presenta mayor frecuencia en pacientes de sexo masculino, de 59,3\% (14), 69,2\% (1), $73,5 \%$ (16) y hasta $85,1 \%$ (13). De igual forma, estas investigaciones muestran mayor pre- valencia de casos en zonas urbanas, hasta en un $68,1 \%$ (13). Como lo reporta la literatura científica, la exposición ocupacional al ambiente agropecuario es uno de los principales factores de riesgo para el desarrollo de la leptospirosis, en nuestro estudio el principal oficio 
relacionado con la leptospirosis fue el agropecuario. Otros estudios colombianos han reportado prevalencias de ocupación agropecuaria bajas, de $6,4 \%$ (1) y $10.3 \%$ (16), justificadas por la mayor cantidad de población urbana en comparación con la rural.

Adicionalmente, el contacto con animales se encuentra altamente relacionado por la infección de este microorganismo, dentro del cual se incluye el contacto con ratas. Este hallazgo es compartido con otros estudios, donde reportan casos que tenían contacto previo con animales en un $64,6 \%$ (15), 81,6\% (16), 83,1\% (14) y hasta un $95,7 \%$ (13).

Esta y otras investigaciones desarrolladas en Colombia coinciden en la presentación clínica de la enfermedad (Tabla 3). El signo más frecuente de la leptospirosis en este estudio fue la fiebre, encontrada en más del $90 \%$ de los pacientes, mientras que los menos frecuentes fueron la hepatomegalia y la ictericia, signos de un compromiso sistémico mayor, el cual ocurre en menor proporción.

La mediana de duración de síntomas fue de seis días. Se trató de un cuadro febril agudo con un retador enfoque diferencial frente a otras entidades infecciosas prevalentes en la región, las cuales se deben tener en cuenta para el manejo específico del paciente.

Este estudio identificó deficiencias en el cumplimiento de las recomendaciones del protocolo de vigilancia en salud pública para leptospirosis, principalmente en la baja proporción de realización de la prueba de tamiz en las IPS y en la toma y remisión de la segunda muestra al laboratorio departamental. Adicionalmente, se encontraron vacíos en el diligenciamiento de la ficha de notificación y en los registros clínicos anexados a esta.

Este estudio corrobora que, dada la limita- da sensibilidad de la prueba de tamiz, todos los casos con sospecha clínica de leptospirosis deben seguir la ruta establecida para hacer confirmación con la prueba de ELISA y, de ser positiva, realizar el MAT, para reducir la proporción y el impacto de los casos que no se diagnostican ni tratan. Es necesario promover la implementación de métodos diagnósticos con mayor rendimiento, a fin de reducir el subdiagnóstico, el subregistro e iniciar tratamiento dirigido al manejo de esta enfermedad, así se evitan desenlaces lamentables para los pacientes. Adicionalmente, se requiere realizar otros estudios a fin de identificar las barreras de acceso diagnóstico y tratamiento de la leptospirosis en el Valle del Cauca, así como explorar los conocimientos de la ruta de confirmación y notificación en el personal de salud.

Durante los dos años estudiados, se evidenció un pico de ocurrencia de la infección por Leptospira en el mes de marzo. Este hallazgo coincide con periodos de lluvia en la región. Además, se evidenció el mayor pico de incidencia durante el mes de mayo del 2018, el cual aportó para ser de este año el de mayor número de casos reportados, y también se relaciona con los periodos de lluvia que se extendieron hasta esa fecha.

La principal limitación de este estudio es el déficit de información en cuanto al reporte obligatorio; se sospecha que exista un subregistro de la enfermedad, tanto en su diagnóstico como en su reporte. Por otra parte, el hecho de no contar con la confirmación microbiológica en todos los casos no permite realizar un análisis frente al rendimiento diagnóstico de las pruebas disponibles. 


\begin{tabular}{|c|c|c|c|c|c|c|c|}
\hline Estudio & $\begin{array}{c}\text { Pacheco et } \\
\text { al. } \\
\text { Valle del } \\
\text { Cauca, } \\
\text { Colombia } \\
2019 \\
(n=105)\end{array}$ & $\begin{array}{l}\text { Pérez.et al. } \\
\text { Urabá, } \\
\text { Colombia } \\
2018 \\
(n=479) \\
(17)\end{array}$ & $\begin{array}{l}\text { Rosso, et al. } \\
\text { Cali, Colombia } \\
\begin{array}{l}2018 \\
(n=87) \\
(16)\end{array}\end{array}$ & $\begin{array}{c}\text { Bello, et al. } \\
\text { Colombia } \\
2013 \\
(n=11786) \\
(11)\end{array}$ & $\begin{array}{c}\text { Echeverri-Toro, } \\
\text { et al } \\
\text { Medellín, } \\
\text { Colombia } \\
2017 \\
(n=119) \\
(18)\end{array}$ & $\begin{array}{l}\text { Tique, et al. } \\
\text { Córdoba, } \\
\text { Colombia } \\
2018 \\
(n=28) \\
(19)\end{array}$ & $\begin{array}{c}\text { Sánchez et al. } \\
\text { Quindío, Colombia } \\
2008 \\
(n=283) \\
(20)\end{array}$ \\
\hline \multicolumn{8}{|c|}{ Información sociodemográfica } \\
\hline $\begin{array}{r}\text { Casos } \\
\text { confirmados }\end{array}$ & & $278(58)$ & & 4621 (31.3) & & - & $69(26.7)$ \\
\hline Sexo masculino & $71(67.6)$ & $186(66.9)$ & $64(73.5)$ & $3196(69.2)$ & $96(79.8)$ & $24(85.7)$ & \\
\hline Zona urbana & $73(69.5)$ & $198(71.4)$ & & $3072(66.5)$ & - & & \\
\hline $\begin{array}{r}\text { Ocupación } \\
\text { agropecuaria }\end{array}$ & $25(23.8)$ & $40(14.3)$ & $9(10.3)$ & $286(6.4)$ & - & $6(21.4)$ & $17(24.6)$ \\
\hline $\begin{array}{r}\text { Contacto con } \\
\text { animales }\end{array}$ & $83(79)$ & & $71(81.6)$ & & - & - & $24(34.6)$ \\
\hline $\begin{array}{r}\text { Contacto con } \\
\text { ratas }\end{array}$ & $46(43.8)$ & & $14(16.1)$ & & - & - & \\
\hline \multicolumn{8}{|c|}{ Características clínicas } \\
\hline Fiebre & $97(92.4)$ & & $74(85.1)$ & & $106(89.1)$ & $28(100)$ & $67(97.1)$ \\
\hline Mialgias & $77(73.3)$ & & $50(57.5)$ & & $88(73.9)$ & $16(57.1)$ & $60(86.9)$ \\
\hline Cefalea & $61(58.1)$ & & $35(40.2)$ & & $63(52.9)$ & $20(71.4)$ & $56(81.1)$ \\
\hline Hepatomegalia & $13(12.4)$ & & $11(12.6)$ & & $49(41.2)$ & $13(46.4)$ & - \\
\hline Ictericia & $32(30.5)$ & & $24(27.6)$ & & $74(62.2)$ & $13(46.4)$ & $36(52.1)$ \\
\hline \multicolumn{8}{|l|}{ Desenlaces } \\
\hline Hospitalización & & & $47(54)$ & & - & - & $48(70 \%)$ \\
\hline $\mathrm{UCl}$ & & & $29(33.3)$ & & $19(16)$ & - & - \\
\hline Transfusión & & & $12(13.8)$ & & - & - & \\
\hline Mortalidad & & $6(2.15)$ & $1(1.1)$ & & $6(5)$ & $4(14.2)$ & $6(8.69)$ \\
\hline
\end{tabular}

Tabla 3: Principales características clínicas y demográficas de la infección por Leptospira reportadas en los estudios realizados en Colombia.

\section{Conclusiones}

En conclusión, la leptospirosis continúa siendo una entidad frecuente en el Valle del Cauca, aunque hay limitaciones en el diagnóstico y la notificación. Los hallazgos de este estudio permiten que el personal de atención médica en el Valle del Cauca conozca el perfil clínico y demográfico reciente de pacientes en estudio para leptospirosis, para incluir esta entidad en el enfoque diagnóstico inicial y diferencial de patologías con sintomatología similar que son de alta prevalencia en la región, y que requieren de un diagnóstico preciso para guiar el manejo específico.

\section{Referencias}

(1) Bello S, Rodríguez M, Paredes A, Mendivelso F, Walteros D, Rodríguez F, et al. Comportamiento de la vigilancia epidemiológica de la leptospirosis humana en Colombia, 2007-2011. Biomédica [Internet]. 2013;33(SUPPL.1):153-60.

(2) Picardeau M. Virulence of the zoonotic agent of leptospirosis: still terra incognita? Nat Rev Microbiol [Internet]. 2017;15(5):297-307. Available from: http://dx.doi.org/10.1038/nrmicro. 2017.5

(3) Ho PL, Haake DA, Hartskeerl RA, Marques M V, Menck CFM, Leite 
González et al

LCC, et al. Comparative Genomics of Two Leptospira interrogans Serovars Reveals Novel Insights into Physiology and Pathogenesis Comparative Genomics of Two Leptospira interrogans Serovars Reveals Novel Insights into Physiology and Pathogenesis $\dagger$. J Bacteriol. 2004;186(7):2164-2172.

(4) Ren SX, Fu G, Jiang XG, Zeng $\mathrm{R}$, Miao $\mathrm{YG}, \mathrm{Xu} \mathrm{H}$, et al. Unique physiological and pathogenic features of Leptospira interrogans revealed by whole-genome sequencing. Nature. 2003;422(6934):888-93.

(5) Mwachui MA, Crump L, Hartskeerl R, Zinsstag J, Hattendorf J. Environmental and Behavioural Determinants of Leptospirosis Transmission: A Systematic Review. PLoS Negl Trop Dis. 2015;9(9):1-15.

(6) Guerra MA. Leptospirosis: Public health perspectives. Biologicals [Internet]. 2013;41(5):295-7. Available from: http://dx.doi.org/10.1016/j.biologicals. 2013.06.010

(7) Felzemburgh RDM, Ribeiro GS, Costa F, Reis RB, Hagan JE, Melendez AXTO, et al. Prospective Study of Leptospirosis Transmission in an Urban Slum Community: Role of Poor Environment in Repeated Exposures to the Leptospira Agent. PLoS Negl Trop Dis. 2014;8(5).

(8) Haake DA, Levett PN. Leptospirosis in Humans. In: Current Topics in Microbiology and Immunology [Internet]. 2015. p. 223-50. Available from: http://link.springer.com/10.1007/9783-662-45059-8

(9) Limmathurotsakul D, Turner EL, Wuthiekanun V, Thaipadungpanit J, Suputtamongkol Y, Chierakul W, et al. Fool's gold: Why imperfect reference tests are undermining the evaluation of novel diagnostics: A reevaluation of 5 diagnostic tests for leptospirosis. Clin Infect Dis. 2012;55(3):322-31.

(10) Costa F, Hagan JE, Calcagno J, Kane M, Torgerson P, Martinez-Silveira MS, et al. Global Morbidity and Mortality of Leptospirosis: A Systematic Review. Small PLC, editor. PLoS Negl Trop Dis [Internet]. 2015 Sep 17 [cited 2017 Sep 22];9(9):e0003898. Available from: http://dx.plos.org/10.1371/journal.pntd. 0003898

(11) Torgerson PR, Hagan JE, Costa F, Calcagno J, Kane M, Martinez-Silveira MS, et al. Global Burden of Leptospirosis: Estimated in Terms of Disability Adjusted Life Years. PLoS Negl Trop Dis. 2015;9(10):1-14.

(12) Cuellar N. Protocolo de vigilancia en Salud Pública. 2015;1-43. Available from: http://manizalessalud.com/wpcontent/uploads/2015/06/PRO-VIHSIDA-Version-3.-Marzo-2015.pdf

(13) Astudillo Hernández M, González Rodríguez A, Batista Santiesteban N, Mirabal Sosa M, Menéndez Hernández J. Estudio seroepidemiológico de la leptospirosis humana en el departamento del Valle del Cauca, 
Colombia. Rev Cuba Med Trop [Internet]. 2009;61(2):1-10. Available from: http://scielo.sld.cu/scielo.php?script= sci_arttext\&amp \%5Cnpid=S0375-

07602009000200004

(14) Ferro BE, Rodríguez AL, Pérez M, Travi BL. Seroprevalencia de infección por Leptospira en habitantes de barrios periféricos de Cali. Biomédica [Internet]. 2006;26(2):250-7. Available from: http://www.ins.gov.co/publicaciones/ 2006_biomedica_262_10_Seroprevalencia_ de_infeccion_por_leptospira.pdf

(15) Osorio L. Seroprevalencia y factores asociados a la infección por Leptospira en un distrito urbano de Cali, Colombia. 2017;33(5):1-14.

(16) Cedano J, Rodríguez S, Kujundzic W, Arana JS, Pacheco R, Rosso F. Caracterización clínica de la leptospirosis grave en un hospital de alta complejidad en Cali, Colombia, 2010-2016. Biomédica [Internet]. 2019;39:108-16. Available from: https://revistabiomedica.org/index.php /biomedica/article/view/3985 